\title{
Ehlers symmetry in four dimensions
}

\author{
Sucheta Majumdar®e \\ Université Libre de Bruxelles and International Solvay Institutes, \\ Campus Plaine - CP 231, B-1050 Bruxelles, Belgium
}

(Received 25 July 2019; published 30 January 2020)

\begin{abstract}
Starting with the light-cone Hamiltonian for gravity, we perform a field redefinition that reveals a hidden symmetry in four dimensions, namely the Ehlers $\operatorname{SL}(2, \mathbb{R})$ symmetry. The field redefinition, which is nonlocal in space but local in time, acts as a canonical transformation in the Hamiltonian formulation keeping the Poisson bracket relations unaltered. We discuss the electromagnetic duality symmetry of gravity in the light-cone formalism, which forms the $S O(2)$ subgroup of this Ehlers symmetry. The helicity states in the original Hamiltonian are not in a representation of the enhanced symmetry group. In order to make the symmetry manifest, we make a change of variables in the path integral from the helicity states to new fields that transform linearly under the $S O(2)$ duality symmetry.
\end{abstract}

DOI: 10.1103/PhysRevD.101.024052

\section{INTRODUCTION}

Theories of gravity and supergravity have been known to exhibit rich hidden symmetries upon dimensional reduction from their higher-dimensional parent theories. Some of the celebrated examples include the Ehlers symmetry and the infinite-dimensional Geroch group in Einstein's gravity $[1,2]$ and the exceptional symmetries in maximal supergravity theories [3-5]. These hidden symmetries are believed to appear when the large spacetime symmetry group in the parent theory splits into a smaller one in lower dimensions, along with some internal symmetries that can further be enhanced using electromagnetic duality.

In recent years, some of these symmetries have been realized in the parent theory prior to dimensional reduction [6-9]. This offers a radically different perspective that these enhanced symmetries might already exist in the higherdimensional parent theories, but may not manifest themselves in a local and Lorentz-covariant formulation. One can choose to make either the spacetime symmetries or the internal symmetries manifest at the level of the action. The light-cone formalism, where Lorentz covariance is not manifest, serves as a suitable platform to look for these symmetries in higher-dimensional theories. The light-cone approach has, in fact, proved to be fruitful for maximal supergravity theories, where we have found strong indications for an $E_{7(7)}$ symmetry in eleven dimensions [10]

Published by the American Physical Society under the terms of the Creative Commons Attribution 4.0 International license. Further distribution of this work must maintain attribution to the author(s) and the published article's title, journal citation, and DOI. Funded by SCOAP ${ }^{3}$. and an $E_{8(8)}$ symmetry in four dimensions [11] up to a nontrivial order in the perturbation constant.

When applied to Einstein's gravity, this approach led to a similar result for the Ehlers symmetry in four dimensions [12], which is originally present only in three dimensions. However, what remained to be understood was how this version of light-cone gravity with a hidden $S L(2, \mathbb{R})$ symmetry could be related to the original light-cone gauge-fixed Einstein-Hilbert action in four dimensions $[13,14]$. In this paper, we attempt to bridge this gap with the help of a field redefinition, which can be interpreted as a canonical transformation in the Hamiltonian formulation. The field redefinition involves some new operators that are nonlocal in space but local in time. The nonlocality occurs only in the field redefinition and not at the level of the action. At a given order in the coupling constant, it can be conjectured that the nonlocal terms so generated can be systematically eliminated by adding correction terms to the field redefinition. We show this explicitly to the second order in the coupling constant.

It is worthwhile to pause and contemplate why the issue of finding the correct field redefinition in the light-cone formalism is interesting. Although we set out to address a very specific problem in light-cone gravity, our motivation is to understand the origin of hidden symmetries in gravity and supergravity theories from a more fundamental standpoint. With the simple example of Ehlers symmetry, we propose a method that takes a given theory to a different formulation, where one can see a symmetry enhancement without any dimensional reduction and subsequent "oxidation" back to four dimensions. The central piece of the puzzle is the $S O(2)$ subgroup of the $S L(2, \mathbb{R})$, that represents the electromagnetic duality symmetry in gravity. The original light-cone action for gravity in four 
dimensions is expressed in terms of the two helicity states of the graviton, which are not suitable for representing the Ehlers symmetry. We must perform a field redefinition that maps these helicity states to new fields that transform linearly under the $S O(2)$ duality group. Thus, our key result is that one must abandon the helicity-invariant configuration in favor of a duality-invariant one, in order to make the Ehlers symmetry manifest in four dimensions.

The paper is organized as follows. In Sec. II we briefly review the work done in [12], where the Ehlers symmetry was realized in light-cone gravity in four dimensions using the tools of dimensional reduction. In Sec. III we present the nonlocal field redefinition that directly takes light-cone gravity in four dimensions to the $S L(2, \mathbb{R})$ invariant version. In Sec. IV, we show that the field redefinition is a canonical transformation in the phase space with the correct Poisson brackets. In Sec. V we present the lightcone representation of the Ehlers symmetry algebra and prove the invariance of the Hamiltonian under this symmetry. We then discuss the duality symmetry in light-cone gravity and explain how its action differs from the little group transformations in four dimensions. We conclude with a few remarks about the relevance of our results to the more sophisticated frameworks, such as the prepotential formalism, exceptional field theories, etc., which explore these symmetry structures at greater lengths.

\section{EHLERS SYMMETRY IN LIGHT-CONE GRAVITY}

We work with the Minkowski signature $\eta^{\mu \nu}=$ $\operatorname{diag}(-1,1,1,1)$. The light-cone coordinates and derivatives are defined as

$$
x^{ \pm}=\frac{x^{0} \pm x^{3}}{\sqrt{2}}, \quad \partial_{ \pm}=\frac{\partial_{0} \pm \partial_{3}}{\sqrt{2}},
$$

where $x^{+}$is considered to be the time coordinate and $\partial_{+}$the time derivative. The transverse coordinates and derivatives are defined as

$$
x=\frac{x^{1}+i x^{2}}{\sqrt{2}}, \quad \bar{x}=\frac{x^{1}-i x^{2}}{\sqrt{2}}
$$

and

$$
\partial=\frac{\partial_{1}+i \partial_{2}}{\sqrt{2}}, \quad \bar{\partial}=\frac{\partial_{1}-i \partial_{2}}{\sqrt{2}}
$$

such that

$$
\bar{\partial} x=\partial \bar{x}=1 .
$$

The light-cone gauge-fixing of the Einstein-Hilbert action is presented in Appendix A. In a perturbative expansion in the coupling constant $\kappa$, the light-cone action for gravity in $d=4$ reads [15]

$$
\begin{aligned}
\mathcal{S}= & \int d^{4} x\left\{\frac{1}{2} \bar{h} \square h+2 \kappa \bar{h} \partial_{-}^{2}\left(-h \frac{\bar{\partial}^{2}}{\partial_{-}^{2}} h+\frac{\bar{\partial}}{\partial_{-}} h \frac{\bar{\partial}}{\partial_{-}} h\right)\right. \\
& \left.+2 \kappa h \partial_{-}^{2}\left(-\bar{h} \frac{\partial^{2}}{\partial_{-}^{2}} \bar{h}+\frac{\partial}{\partial_{-}} \bar{h} \frac{\partial}{\partial_{-}} \bar{h}\right)+O\left(\kappa^{2}\right)\right\},
\end{aligned}
$$

where $h$ and $\bar{h}$ are the two helicity states of the graviton. Upon dimensional reduction, Einstein's gravity in three dimensions exhibits an $S L(2, R)$ symmetry due to Ehlers [1], which acts like a nonlinear sigma-model symmetry on these fields

$$
\delta h=\frac{1}{\kappa}(\text { constant })+\kappa(\text { quadratic in field })+\cdots .
$$

We now briefly discuss the Ehlers symmetry in the lightcone gauge and how it can be realized in four dimensions in the following three steps. The details can be found in [12].

Step 1: Dimensional reduction.-We obtain the action for light-cone gravity in three dimensions by removing the dependence of the fields in one of the transverse coordinates, namely $x^{2}$. In other words, we set the transverse derivative $\bar{\partial}=\partial$ in the action (2.3).

Step 2: Field redefintion.- - In order to look for a nonlinear sigma model symmetry of the form (2.4), we first have to eliminate the cubic interaction vertices from the three-dimensional action. One way to understand this is to consider the Ehlers symmetry as a remnant of the $E_{8(8)}$ symmetry in the maximal supergravity theory in three dimensions. The $E_{8(8)}$ symmetry in $d=3$ can be decomposed into a linear $S O(16)$ part and a nonlinear coset as

$$
E_{8(8)}=S O(16) \times \frac{E_{8(8)}}{S O(16)}
$$

which, upon supersymmetric truncation to pure gravity, yields

$$
S L(2, \mathbb{R})=S O(2) \times \frac{S L(2, \mathbb{R})}{S O(2)} .
$$

However, the $S O(16)$ part of the $E_{8(8)}$ symmetry does not admit any interaction vertices of odd-order $\left(\kappa, \kappa^{3}, \ldots\right)$ in the action. We, therefore, remove the three-point interaction terms obtained from the dimensional reduction of (2.3) through the following field redefinition:

$$
\begin{aligned}
h \rightarrow & h^{\prime}-\kappa \partial_{-}^{2}\left(\frac{1}{\partial_{-}} h^{\prime} \frac{1}{\partial_{-}} h^{\prime}\right)-2 \kappa \frac{1}{\partial_{-}^{2}}\left(\partial_{-}^{3} h^{\prime} \frac{1}{\partial_{-}} \bar{h}^{\prime}\right) \\
& +\mathcal{O}\left(\kappa^{2}\right) .
\end{aligned}
$$

The field redefinition gives rise to some new interaction terms in the action involving time derivatives, which can be further eliminated by adding correction terms to (2.7). The action so obtained is invariant under the following set of transformations: 


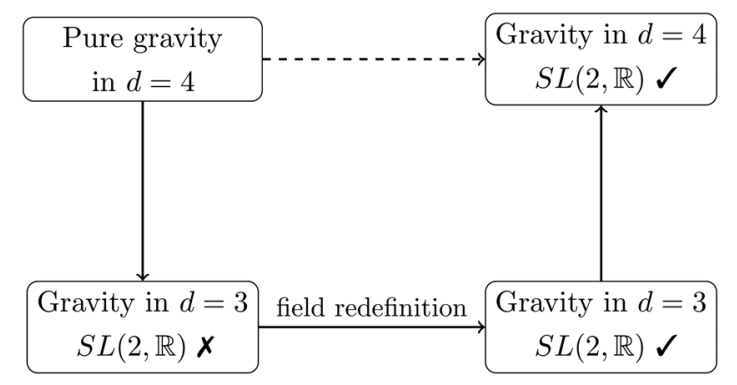

FIG. 1. Ehlers symmetry in $d=4$ gravity.

$L_{+} h^{\prime}=\frac{1}{\kappa} a-\kappa a \frac{1}{\partial_{-}}\left(\partial_{-} h^{\prime} \bar{h}^{\prime}\right)-2 \kappa a \frac{1}{\partial_{-}^{2}}\left(\partial_{-}^{3} h^{\prime} \frac{1}{\partial_{-}} \bar{h}^{\prime}\right)$,

$L_{-} h^{\prime}=-2 \kappa \bar{a} \frac{1}{\partial_{-}^{2}}\left(\partial_{-}^{3} h^{\prime} \frac{1}{\partial_{-}} \bar{h}^{\prime}\right)+\frac{1}{2} \kappa \bar{a} h^{\prime} h^{\prime}$,

with the conjugate expressions for $\bar{h}^{\prime}$. These transformations, which satisfy an $S L(2, \mathbb{R})$ algebra, constitute the light-cone representation of the Ehlers symmetry in three dimensions. The new fields $h^{\prime}$ and $\bar{h}^{\prime}$ can be treated as scalars in three dimensions, which parametrize the $S L(2, \mathbb{R}) / S O(2)$ coset, the same way as the 128 scalars in $\mathcal{N}=16$ supergravity parametrize the $E_{8(8)} / S O(16)$ coset.

Step 3: Dimensional "oxidation".-We can now perform a dimensional uplift by reintroducing the $x^{2}$ coordinate following the prescription in [16]. The idea is to define a "generalized derivative" of the form

$$
\nabla=a \partial_{1}+b \partial_{2}
$$

and use the Poincare algebra in four dimensions to fix the coefficients $a$ and $b$. We can then invoke the quadratic form property of the Hamiltonian [17] and modify the derivative structure of the four-dimensional Hamiltonian using $\nabla$. We can, thus, "oxidize" the theory to four dimensions keeping the Ehlers symmetry preserved in a way that is consistent with the Poincaré symmetry.

We therefore arrive at a theory of light-cone gravity in $d=4$ with a hidden $S L(2, \mathbb{R})$ symmetry. It appears as though there exist two distinct formulations of light-cone gravity in four dimensions with different symmetry structures, as depicted in Fig. 1.

Our goal in this paper is to formulate a path from the original $d=4$ light-cone theory of gravity to the $S L(2, \mathbb{R})$ invariant version, without having to go through the longer route involving dimensional reduction and "oxidation". Such a path will connect the two different formulations of gravity in four dimensions and thereby serve as the missing link (dashed arrow) in the figure above.

\section{NONLOCAL FIELD REDEFINITION IN $\boldsymbol{d}=\mathbf{4}$}

The field redefinition from light-cone gravity to the $S L(2, \mathbb{R})$ invariant version in four dimensions requires some new nonlocal operators. Before we define these operators, let us consider the derivative structure of the light-cone Hamiltonian in $d=4$

$$
\begin{aligned}
H \sim & \partial \bar{h} \bar{\partial} h+\kappa(\bar{\partial})^{2}(h)^{2} \bar{h}+\kappa(\partial)^{2}(\bar{h})^{2} h+\kappa^{2}(\partial \bar{\partial})(h)^{2}(\bar{h})^{2} \\
& +\cdots
\end{aligned}
$$

to demonstrate why we need to introduce them in the first place. In order to find a field redefinition that absorbs the cubic interaction vertices into the kinetic term, we have to define an operation which "converts" a $\partial$ into a $\bar{\partial}$ and vice versa. In the light-cone formalism, we use the $\frac{1}{\partial_{-}}$operator liberally to achieve the effect of removing a $\partial_{-}$derivative. We shall now define similar operators with the other two spatial derivatives.

\section{A. Nonlocal operators}

In light-cone field theories, the $\frac{1}{\partial_{-}}$operator is defined in terms of the Heaviside step function $[14,18]$. Consider two functions $g\left(x^{-}\right)$and $f\left(x^{-}\right)$such that

$$
\partial_{-} g\left(x^{-}\right)=f\left(x^{-}\right)
$$

The $\frac{1}{\partial_{-}}$operator allows us to solve for $g\left(x^{-}\right)$up to an arbitrary function $h$ independent of $x^{-}$

$g\left(x^{-}\right)=\frac{1}{\partial_{-}} f\left(x^{-}\right)=\int_{-\infty}^{\infty} \epsilon\left(x^{-}-y^{-}\right) f\left(y^{-}\right) d y^{-}+h$.

The function $h$ can further be removed by imposing appropriate boundary conditions. ${ }^{1}$ This integral operator is nonlocal in the $x^{-}$coordinate by construction. The function $f\left(x^{-}\right)$can be recovered by acting with a $\partial_{-}$derivative on both sides of the above equation

$$
\partial_{-} g\left(x^{-}\right)=\partial_{-} \frac{1}{\partial_{-}} f\left(x^{-}\right)=f\left(x^{-}\right)
$$

One also allows for multiple poles which satisfy the relation [14]

$$
\frac{1}{\partial_{-}{ }^{n}} f=(n-1)\left[x^{-}, \frac{1}{\partial_{-}^{n-1}}\right] f .
$$

We now extend this "inverse of a derivative" operation to the other two spatial coordinates. We define new operators

$$
\frac{1}{\partial} f(\bar{x})=\int_{-\infty}^{\infty} \epsilon(\bar{x}-\bar{y}) f(\bar{y}) d \bar{y}
$$

\footnotetext{
${ }^{1}$ We have imposed asymptotically Minkowski boundary conditions, i.e., $g^{\mu \nu} \rightarrow \eta^{\mu \nu}$ at infinity, which allow us to freely perform partial integration and drop the surface terms.
} 
and

$$
\frac{1}{\bar{\partial}} f(x)=\int_{-\infty}^{\infty} \epsilon(x-y) f(y) d y,
$$

that are local in time $x^{+}$but nonlocal in the $\bar{x}$ and $x$ coordinates respectively. In the momentum space one can define suitable pole prescriptions for $p, \bar{p} \rightarrow 0$ following [18], which are relevant for loop calculations in the quantum theory. ${ }^{2}$ For the purpose of this paper, however, it suffices to treat these operators formally in the same fashion as in (3.4), namely

$$
\partial \frac{1}{\partial} f(\bar{x})=f(\bar{x}), \quad \bar{\partial} \frac{1}{\bar{\partial}} f(x)=f(x) .
$$

In the next section, we discuss a field redefinition which eliminates the cubic interaction vertices from the light-cone Hamiltonian by making use of these new operators.

\section{B. The field redefinition}

We work in a Hamiltonian framework starting with the light-cone Hamiltonian for gravity in $d=4$, which reads [15]

$$
\begin{aligned}
\mathcal{H}= & \partial \bar{h} \bar{\partial} h-2 \kappa \bar{h} \partial_{-}^{2}\left(\frac{\bar{\partial}}{\partial_{-}} h \frac{\bar{\partial}}{\partial_{-}} h-h \frac{\bar{\partial}^{2}}{\partial_{-}^{2}} h\right)-2 \kappa h \partial_{-}^{2}\left(\frac{\partial}{\partial_{-}} \bar{h} \frac{\partial}{\partial_{-}} \bar{h}-\bar{h} \frac{\partial^{2}}{\partial_{-}^{2}} \bar{h}\right) \\
& -2 \kappa^{2}\left\{\frac{1}{\partial_{-}^{2}}\left(\partial_{-} h \partial_{-} \bar{h}\right) \frac{\partial \bar{\partial}}{\partial_{-}^{2}}\left(\partial_{-} h \partial_{-} \bar{h}\right)+\frac{1}{\partial_{-}^{3}}\left(\partial_{-} h \partial_{-} \bar{h}\right)\left(\partial \bar{\partial} h \partial_{-} \bar{h}+\partial_{-} h \partial \bar{\partial} \bar{h}\right)\right. \\
& -\frac{1}{\partial_{-}^{2}}\left(\partial_{-} h \partial_{-} \bar{h}\right)\left(2 \partial \bar{\partial} h \bar{h}+2 h \partial \bar{\partial} \bar{h}+9 \bar{\partial} h \partial \bar{h}+\partial h \bar{\partial} \bar{h}-\frac{\partial \bar{\partial}}{\partial_{-}} h \partial_{-} \bar{h}-\partial_{-} h \frac{\partial \bar{\partial}}{\partial_{-}} \bar{h}\right) \\
& -2 \frac{1}{\partial_{-}}\left(2 \bar{\partial} h \partial_{-} \bar{h}+h \partial_{-} \bar{\partial} \bar{h}-\partial_{-} \bar{\partial} h \bar{h}\right) h \partial \bar{h}-2 \frac{1}{\partial_{-}}\left(2 \partial_{-} h \partial \bar{h}+\partial_{-} \partial h \bar{h}-h \partial_{-} \partial \bar{h}\right) \bar{\partial} h \bar{h} \\
& -\frac{1}{\partial_{-}}\left(2 \bar{\partial} h \partial_{-} \bar{h}+h \partial_{-} \bar{\partial} \bar{h}-\partial_{-} \bar{\partial} h \bar{h}\right) \frac{1}{\partial_{-}}\left(2 \partial_{-} h \partial \bar{h}+\partial_{-} \partial h \bar{h}-h \partial_{-} \partial \bar{h}\right) \\
& \left.-h \bar{h}\left(\partial \bar{\partial} h \bar{h}+h \partial \bar{\partial} \bar{h}+2 \bar{\partial} h \partial \bar{h}+3 \frac{\partial \bar{\partial}}{\partial_{-}} h \partial_{-} \bar{h}+3 \partial_{-} h \frac{\partial \bar{\partial}}{\partial_{-}} \bar{h}\right)\right\}+\mathcal{O}\left(\kappa^{3}\right) .
\end{aligned}
$$

In order to eliminate the three-point interaction vertices in the Hamiltonian, we perform the field redefinition

$$
h=C-\kappa \frac{\bar{\partial}}{\partial} \partial_{-}^{2}\left(\frac{1}{\partial_{-}} C \frac{1}{\partial_{-}} C\right)-2 \kappa \frac{\partial}{\bar{\partial}} \frac{1}{\partial_{-}^{2}}\left(\partial_{-}^{3} C \frac{1}{\partial_{-}} \bar{C}\right),
$$

which brings the Hamiltonian to the form

$$
\mathcal{H}^{\prime}=\partial \bar{C} \bar{\partial} C+\mathcal{O}\left(\kappa^{2}\right) .
$$

The $\frac{1}{\partial}$ and $\frac{1}{\partial}$ operators generate new terms in the Hamiltonian at order $\kappa^{2}$, which are nonlocal in the $x$ and $\bar{x}$ coordinates. These terms can be removed by adding some $\kappa^{2}$-correction terms to (3.10)

$$
\begin{aligned}
h= & C-\kappa \frac{\bar{\partial}}{\partial} \partial_{-}^{2}\left(\frac{1}{\partial_{-}} C \frac{1}{\partial_{-}} C\right)-2 \kappa \frac{\partial}{\bar{\partial}} \frac{1}{\partial_{-}^{2}}\left(\partial_{-}^{3} C \frac{1}{\partial_{-}} \bar{C}\right) \\
& -2 \kappa^{2} \frac{1}{\bar{\partial} \partial_{-}}\left[\frac{\partial}{\partial_{-}} \bar{C} \frac{\bar{\partial}}{\partial} \partial_{-}^{4}\left(\frac{1}{\partial_{-}} C \frac{1}{\partial_{-}} C\right)\right]+2 \kappa^{2} \frac{\partial}{\bar{\partial} \partial_{-}^{2}}\left[C \frac{\bar{\partial}}{\partial} \partial_{-}^{4}\left(\frac{1}{\partial_{-}} C \frac{1}{\partial_{-}} C\right)\right] \\
& +4 \kappa^{2} \frac{\partial}{\bar{\partial} \partial_{-}^{2}}\left[\partial_{-}^{2} C \frac{\bar{\partial}}{\partial \partial_{-}^{2}}\left(\partial_{-}^{3} \bar{C} \frac{1}{\partial_{-}} C\right)\right]+4 \kappa^{2} \frac{\partial}{\bar{\partial} \partial_{-}^{2}}\left[\bar{C} \frac{\partial}{\bar{\partial}}\left(\partial_{-}^{3} C \frac{1}{\partial_{-}} \bar{C}\right)\right] \\
& -4 \kappa^{2} \frac{1}{\bar{\partial} \partial_{-}}\left[\partial_{-}^{2} C \frac{\partial^{2}}{\bar{\partial}} \partial_{-}\left(\frac{1}{\partial_{-}} \bar{C} \frac{1}{\partial_{-}} \bar{C}\right)\right]+4 \kappa^{2} \frac{1}{\bar{\partial} \partial_{-}}\left[\frac{\partial^{2}}{\bar{\partial}}\left(\bar{C} \partial_{-}^{2} C\right) \frac{1}{\partial_{-}} \bar{C}\right] \\
& +2 \kappa^{2} \frac{\partial}{\bar{\partial} \partial_{-}^{2}}\left[\partial_{-}^{2} C \frac{\partial}{\bar{\partial}} \partial_{-}^{2}\left(\frac{1}{\partial_{-}} C \frac{1}{\partial_{-}} C\right)\right] .
\end{aligned}
$$

\footnotetext{
${ }^{2}$ One encounters a similar kind of nonlocality in the momentum space, while constructing maximally helicity violating Lagrangians for Yang-Mills theory and gravity using field redefinitions [15,19].
} 
Thus, one can ensure that the Hamiltonian to order $\kappa^{2}$ does not involve any nonlocal terms by adding these correction terms to the field redefinition. We can argue that the above statement holds at any order in the coupling constant, provided that the field redefinition is appropriately modified to that order. The field redefinition also generates some local terms ${ }^{3}$ which we add to the four-point vertex in the new Hamiltonian, which now reads

$$
\begin{aligned}
\mathcal{H}^{\prime}= & \bar{\partial} C \partial \bar{C}+\mathcal{H}^{\kappa^{2}}+\kappa^{2} \partial \partial_{-}^{2}\left(\frac{1}{\partial_{-}} \bar{C} \frac{1}{\partial_{-}} \bar{C}\right) \bar{\partial} \partial_{-}^{2}\left(\frac{1}{\partial_{-}} C \frac{1}{\partial_{-}} C\right) \\
& +4 \kappa^{2} \frac{\bar{\partial}}{\partial_{-}^{2}}\left(\partial_{-}^{3} \bar{C} \frac{1}{\partial_{-}} C\right) \frac{\partial}{\partial_{-}^{2}}\left(\partial_{-}^{3} C \frac{1}{\partial_{-}} \bar{C}\right) \\
& +4 \kappa^{2}\left\{2 \partial_{-}^{2} \bar{C} \frac{\partial}{\partial_{-}^{3}}\left(\partial_{-}^{3} C \frac{1}{\partial_{-}} \bar{C}\right) \frac{\bar{\partial}}{\partial_{-}} C-\partial_{-}^{2} \bar{C} C \frac{\partial \bar{\partial}}{\partial_{-}^{4}}\left(\partial_{-}^{3} C \frac{1}{\partial_{-}} \bar{C}\right)+\text { c.c. }\right\} .
\end{aligned}
$$

Here $\mathcal{H}^{\kappa^{2}}$ on the right-hand side stands for the $\kappa^{2}$-interaction terms obtained from the old Hamiltonian (3.9) with $h$ replaced by $C$ at the lowest order. Before we proceed to discuss the symmetries of the new Hamiltonian, we first study the role this field redefinition plays in the Hamiltonian formulation.

\section{THE FIELD REDEFINITION AS A CANONICAL TRANSFORMATION}

In this section, we demonstrate how the nonlocal field redefinition can be viewed as an infinitesimal canonical transformation in the phase space. Henceforth we treat the Hamiltonian (3.9) as the fundamental entity and the Lagrangian is always derived from it using a Legendre transform $^{4}$

$$
\mathcal{L}=\pi_{h} \partial_{+} h+\pi_{\bar{h}} \partial_{+} \bar{h}-\mathcal{H},
$$

$\pi_{h}$ and $\pi_{\bar{h}}$ being the momenta conjugate $h$ and $\bar{h}$ respectively. The phase space variables $\left(h, \bar{h}, \pi_{h}, \pi_{\bar{h}}\right)$ satisfy the following Poisson bracket relations:

$$
\begin{gathered}
\{h(x), \bar{h}(y)\}=\{h(x), h(y)\}=\{\bar{h}(x), \bar{h}(y)\}=0, \\
\left\{\pi_{h}(x), \pi_{\bar{h}}(y)\right\}=\left\{\pi_{h}(x), \pi_{h}(y)\right\}=\left\{\pi_{\bar{h}}(x), \pi_{\bar{h}}(y)\right\}=0 \\
\left\{h(x), \pi_{h}(y)\right\}=\left\{\bar{h}(x), \pi_{\bar{h}}(y)\right\}=\delta^{(3)}(x-y),
\end{gathered}
$$

\footnotetext{
${ }^{3}$ By local terms, we mean the terms that do not have any nonlocality other than the $\frac{1}{\partial_{-}}$type and in particular, do not involve any $\frac{1}{\partial}$ or $\frac{1}{\partial}$ operators.

${ }^{4}$ From our knowledge of $\mathcal{L}(2.3)$, we can define the conjugate momenta as

$$
\pi_{h}=\frac{\delta \mathcal{L}}{\delta\left(\partial_{+} h\right)}=\partial_{-} \bar{h} .
$$

But as we are working in the Hamiltonian formulation, we treat the conjugate momenta as independent variables and do not need to identify $\pi_{h}$ with $\partial_{-} \bar{h}$ or even define it in terms of $\mathcal{L}$.
}

where the Poisson brackets with $h, \pi_{h}$ are defined as

$$
\{A, B\}_{h, \pi_{h}}=\left(\frac{\delta A}{\delta h} \frac{\delta B}{\delta \pi_{h}}-\frac{\delta B}{\delta h} \frac{\delta A}{\delta \pi_{h}}\right)
$$

and similarly with $\bar{h}, \pi_{\bar{h}}$. (The implicit dependence on spacetime coordinates has been supressed for notational simplicity.)

To construct the Lagrangian corresponding to the "new" Hamiltonian (3.13)

$$
\mathcal{L}^{\prime}=\pi_{C} \partial_{+} C+\pi_{\bar{C}} \partial_{+} \bar{C}-\mathcal{H}^{\prime},
$$

we need to suitably define the conjugate momenta $\pi_{C}$ and $\pi_{\bar{C}}$ in order that the new fields satisfy the correct Poisson bracket relations as before, for e.g.,

$$
\left\{C(x), \pi_{C}(y)\right\}=\left\{\bar{C}(x), \pi_{\bar{C}}(y)\right\}=\delta^{(3)}(x-y) .
$$

This is guaranteed if we can show that the field redefinition (3.10) from $(h, \bar{h})$ to $(C, \bar{C})$ is just an infinitesimal canonical transformation in the phase space leaving the Poisson brackets invariant, i.e.,

$$
\left\{h(x), \pi_{h}(y)\right\}_{h, \pi_{h}}=\left\{C(x), \pi_{C}(y)\right\}_{h, \pi_{h}}=\delta^{(3)}(x-y) .
$$

\section{A. The generating functional}

We construct a generating functional of type 3 involving the new fields $C, \bar{C}$ and the old momenta $\pi_{h}, \pi_{\bar{h}}$ following the discussion in [15]

$G\left(C, \bar{C}, \pi_{h}, \pi_{\bar{h}}\right)=\int g(C, \bar{C}) \pi_{h}+\int \bar{g}(C, \bar{C}) \pi_{\bar{h}}$,

where we choose the function $g(C, \bar{C})$ to be

$$
\begin{aligned}
g(C)= & C-\kappa \frac{\bar{\partial}}{\partial} \partial_{-}^{2}\left(\frac{1}{\partial_{-}} C \frac{1}{\partial_{-}} C\right)-2 \kappa \frac{\partial}{\bar{\partial}} \frac{1}{\partial_{-}^{2}}\left(\partial_{-}^{3} C \frac{1}{\partial_{-}} \bar{C}\right) \\
& +\mathcal{O}\left(\kappa^{2}\right) .
\end{aligned}
$$


We can derive the rest of the variables, namely the old fields and new momenta, from the generating functional $G$. The old field variables $h, \bar{h}$ can be derived from the generating functional as

$$
h=\frac{\delta G}{\delta \pi_{h}}=g(C, \bar{C}), \quad \bar{h}=\frac{\delta G}{\delta \pi_{\bar{h}}}=\bar{g}(C, \bar{C}),
$$

which reproduce our desired field redefinition (3.10) as a canonical transformation. The new conjugate momentum $\pi_{C}$ is defined as

$$
\begin{aligned}
\pi_{C} & =\frac{\delta G}{\delta C}=\int \frac{\delta g}{\delta C} \pi_{h}+\int \frac{\delta \bar{g}}{\delta \bar{C}} \pi_{\bar{h}} \\
& =\int d^{3} x \pi_{h}(x) \frac{\delta h(x)}{\delta C(y)}+\int d^{3} x \pi_{\bar{h}}(x) \frac{\delta \bar{h}(x)}{\delta \bar{C}(y)},
\end{aligned}
$$

where we have used the fact that $h=g(C, \bar{C})$. Similarly $\pi_{\bar{C}}$ can be calculated from (4.10) as the functional derivative of $G$ with respect to $\bar{C}$.

We can now verify that the canonical transformation generated by the functional $G$ preserves the Poisson bracket relations. We illustrate one such relation below. [Note the second term in (4.12) does not contribute in this case.]

$$
\begin{aligned}
& \left\{C(x), \pi_{C}(y)\right\}_{h, \pi_{h}} \\
& =\left(\frac{\delta C(x)}{\delta h(z)} \frac{\delta \pi_{C}(y)}{\delta \pi_{h}(z)}-\frac{\delta C(x)}{\delta \pi_{h}(z)} \frac{\delta \pi_{C}(y)}{\delta h(z)}\right) \\
& =\frac{\delta C(x)}{\delta h(z)} \frac{\delta}{\delta \pi_{h}(z)}\left\{\int d^{3} y^{\prime} \pi_{h}\left(y^{\prime}\right) \frac{\delta h\left(y^{\prime}\right)}{\delta C(y)}\right\}-0 \\
& =\int d^{3} y^{\prime} \frac{\delta C(x)}{\delta h(z)} \delta^{(3)}\left(z-y^{\prime}\right) \frac{\delta h\left(y^{\prime}\right)}{\delta C(y)} \\
& =\frac{\delta C(x)}{\delta h(z)} \frac{\delta h(z)}{\delta C(y)}=\delta^{(3)}(x-y) .
\end{aligned}
$$

One could argue that the above discussion holds true for any form of $G$ and hence, for any change of variables. Indeed the crucial point here is that even in the case where the change of variables involves some nonlocal operators, one can interpret it as a canonical transformation in the Hamiltonian formulation.

Therefore, the canonical transformation (2.7) maps the original light-cone Hamiltonian to the new Hamiltonian without any cubic vertices, which now describes the theory in terms of the fields $(C, \bar{C})$. The field redefinition merely amounts to a change of variables in the path integral as discussed in Appendix B.

The crux of the problem is that the helicity states $(h, \bar{h})$ in the original action are not the "good" field variables for representing the enhanced symmetry. We must make a change of variables to the appropriate fields $(C, \bar{C})$ to make the symmetry manifest. Having shown that the two formulations describe the same theory, we can now proceed to investigate the symmetries the new Hamiltonian exhibits, which are obscured in the $h, \bar{h}$ configuration.

\section{EHLERS SYMMETRY IN $d=4$}

To find the Ehlers symmetry in four dimensions, we start with a sigma-model-like transformation with parameters $a$ and $\bar{a}$

$$
\delta C=\frac{1}{\kappa} a, \quad \delta \bar{C}=\frac{1}{\kappa} \bar{a}
$$

and consider the variation of the new Hamiltonian (3.13) under these transformations

$$
\mathcal{H}^{\prime}=\mathcal{H}^{\prime 0}+\mathcal{H}^{\prime \kappa^{2}}=\bar{\partial} C \partial \bar{C}+\kappa^{2}(\cdots) .
$$

At the lowest order, the Hamiltonian varies as

$$
\left(\delta \mathcal{H}^{\prime}\right)^{\kappa^{-1}}=\bar{\partial}(\delta C) \partial \bar{C}+\bar{\partial} C \partial(\delta \bar{C}),
$$

which vanishes trivially since the parameters $a$ and $\bar{a}$ are constants. At the next order, we have

$$
\left(\delta \mathcal{H}^{\prime}\right)^{\kappa}=\delta^{\kappa} \mathcal{H}^{\prime 0}+\delta^{\kappa^{-1}} \mathcal{H}^{\kappa^{2}} .
$$

The variation of the interacting part of the Hamiltonian $\delta^{\kappa^{-1}} \mathcal{H}^{\kappa^{2}}$ can be compensated for by adding correction terms of order $\kappa$ terms to (5.1)

$$
\begin{aligned}
& \delta C=\frac{1}{\kappa} a+2 \kappa a \frac{1}{\partial_{-}}\left(\partial_{-} C \bar{C}\right)-\kappa \bar{a} C C, \\
& \delta \bar{C}=\frac{1}{\kappa} \bar{a}+2 \kappa \bar{a} \frac{1}{\partial_{-}}\left(\partial_{-} \bar{C} C\right)-\kappa a \bar{C} \bar{C},
\end{aligned}
$$

which act on $\mathcal{H}^{\prime 0}$, thereby rendering the Hamiltonian invariant up to order $\kappa^{2}$. Therefore, the transformations (5.3) correspond to a symmetry of the new Hamiltonian.

To reveal the Lie algebra underlying this symmetry, we rewrite these transformations as a set of two transformations parametrized by $a$ and $\bar{a}$ respectively,

$$
\begin{aligned}
L_{+} C & =\frac{1}{\kappa} a+2 \kappa a \frac{1}{\partial_{-}}\left(\partial_{-} C \bar{C}\right), \\
L_{+} \bar{C} & =-\kappa a \bar{C} \bar{C}, \\
L_{-} C & =-\kappa \bar{a} C C, \\
L_{-} \bar{C} & =\frac{1}{\kappa} \bar{a}+2 \kappa \bar{a} \frac{1}{\partial_{-}}\left(\partial_{-} \bar{C} C\right) .
\end{aligned}
$$

We augment the above transformations with an $S O(2)$ transformation acting linearly on the fields

$$
L_{0} C=2 a_{0} C, \quad L_{0} \bar{C}=-2 a_{0} \bar{C} .
$$


We find that these $L_{+}, L_{-}$, and $L_{0}$ transformations satisfy an $\mathrm{SL}(2, \mathrm{R})$ algebra

$$
\left[L_{+}, L_{-}\right]=L_{0}, \quad\left[L_{0}, L_{ \pm}\right]=L_{ \pm} .
$$

Thus, we recover the Ehlers symmetry in $d=4$ light-cone gravity found in [12], without having to reduce the theory to three dimensions. We must remember though that the symmetry is only strictly proven to order $\kappa^{2}$ in the Hamiltonian, but we do not expect any formal difficulties in extending these results to higher orders. However, the explicit calculations become quite intractable to be done analytically since the number of terms at each order increases tremendously.

The $L_{0}$ transformations seem to have been added ad hoc for the algebra to close. These transformations mimic the action of the little group rotations in four dimensions, which assigns helicity values to the fields. On the contrary, this $S O(2)$ is inherently different from the helicity group as we discuss below.

\section{A. $S O(2)$ little group versus duality symmetry}

Little group transformations in four dimensions, which rotate the transverse coordinates and derivatives into each other, are generated by the $J^{12}$ element of the light-cone Poincaré algebra. In the massless case, its spin part $S^{12}$ determines the helicity of the fields [14], for example,

$$
S^{12}\left(\begin{array}{c}
h \\
\bar{h}
\end{array}\right)=2\left(\begin{array}{c}
h \\
-\bar{h}
\end{array}\right) .
$$

The light-cone Lagrangian (2.3) for gravity expressed in terms of these fields is thus helicity invariant, even though Lorentz invariance is no longer manifest. In particular, the cubic interaction vertices are manifestly helicity invariant because the derivatives $\partial$ and $\bar{\partial}$ also transform under the $S O(2)$ with helicity values +1 and -1 respectively.

We now return to the $L_{0}$ transformations, which form the maximal compact subgroup of the $S L(2, \mathbb{R})$ discussed in the last section

$$
L_{0}\left(\begin{array}{l}
C \\
\bar{C}
\end{array}\right)=2 a_{0}\left(\begin{array}{c}
C \\
-\bar{C}
\end{array}\right) .
$$

It seems that $L_{0}$ can be identified with the helicity group (5.8), if we set the parameter $a_{0}$ to identity. But as the $S L(2, \mathbb{R})$ is a hidden symmetry of the theory, the $S O(2)$ subgroup must also be a part of this internal symmetry and not the Poincaré group.

This $S O(2)$ is a remnant of the electromagnetic duality symmetry in gravity, which leaves the equations of motion invariant under the exchange of the electric and magnetic part of the curvature tensor [20]. In the light-cone formalism, since we have fixed the gauge completely and eliminated all the unphysical degrees of freedom (d.o.f.), the equations of motion are not expressed in terms of the curvature, but the fields $C$ and $\bar{C}$. Therefore, the electromagnetic duality symmetry is also translated in terms of rotations in the two remaining d.o.f. of the graviton. The new fields $C$ and $\bar{C}$ are, thus, the eigenstates of the duality operator $L_{0}$, just as the old fields $h$ and $\bar{h}$ are the eigenstates of the helicity operator $S^{12}$. This is in agreement with [20] that the components of the metric tensor are not good variables for constructing a manifestly duality-invariant action; one should instead introduce prepotentials and express the symmetry transformations in terms of an $S O(2)$ rotation acting on the prepotentials.

Unlike the little group transformations, the $S O(2)$ duality does not act on the derivatives $\partial$ and $\bar{\partial}$. As a result, it is not a symmetry of the original light-cone action due to the presence of cubic vertices. To realize this symmetry, we must give up the helicity-invariant formulation in favor of the duality-invariant one, where we have removed the cubic vertices through a suitable field redefinition. This interpretation resonates with the idea that hidden symmetries in gravity and supergravity theories arise from a trade-off between the spacetime symmetries and the internal symmetries.

In our approach the fields depend on all four spacetime coordinates in both the formulations. In spirit, this is similar to the dual graviton and exceptional field theories [6,7], where one considers a $D=n+d$ split in a $D$-dimensional theory with certain constraints to be fulfilled by the $d$ dimensional internal space. The key difference is that their approach is manifestly covariant and local, while ours is not. Nonetheless, the crucial point is that we are not performing any dimensional reduction to uncover the enhanced symmetry in $d=4$, neither are we constraining the dynamics of the theory to a three-dimensional subspace.

\section{CONCLUDING REMARKS}

In the light-cone superspace, $\mathcal{N}=8$ supergravity in four dimensions can be shown to exhibit an $E_{8(8)}$ symmetry, enhanced from the previously known $E_{7(7)}$ symmetry [11]. In order to realize this symmetry enhancement, one must abandon the helicity states, originally in representations of the $S U(8)$ R-symmetry group, in favor of an $S O(16)$ representation which mixes fields of different spins. In [12], the Ehlers symmetry in four dimensions was obtained as a remnant of this $E_{8}$ symmetry in $N=8$ supergravity after supersymmetric truncation to pure gravity. The obvious next step would be to repeat the analysis presented here for the $\mathcal{N}=8$ theory to find a suitable map from the helicity states to the appropriate field variables, on which the $E_{8(8)}$ symmetry can be made manifest in four dimensions.

The important lesson we learn is that only in the right field configuration can we see the various symmetries the theory possesses. In order to find all the symmetries, we 
have to know the correct field redefinitions that allow us to shift between various configurations. This opens the door to many intriguing possibilities. One of the most pressing questions it raises is whether the Ehlers symmetry is the only hidden symmetry one can realize in four-dimensional Einstein's gravity or is there room for more. The immediate candidate for a further symmetry enhancement would be the Geroch group in Einstein's theory. On reduction to two dimensions, the theory has an $S L(2, \mathbb{R})$ symmetry called the Matzner-Misner group [21], which does not commute with the existing Ehlers $S L(2, \mathbb{R})$ group; instead they generate the infinite-dimensional Geroch group. To understand the Geroch group in the light-cone language, first we must find the right formulation to realize the MatznerMisner symmetry. For supergravity theories, one can pose similar questions: Is $E_{8(8)}$ symmetry the largest symmetry group one can realize in four dimensions or can we extend it further to $E_{9}$ and $E_{10}$ ? Can we find a field redefinition in eleven-dimensional supergravity, that maps the theory to a different configuration with manifest $E_{7(7)}$ or $E_{8(8)}$ symmetry?

It has been established time and again that the Hamiltonian formulation is better suited than the Lagrangian formulation for the study of hidden symmetries off shell. In [20], the duality-invariant action for linearzied Einstein's gravity was constructed using prepotentials in the Hamiltonian formulation. We obtain a duality-invariant formulation for light-cone gravity without restricting the theory to the linearized order. Admittedly, our perturbative results are not sufficient to comment on the nature of the full nonlinear theory. Nevertheless, the fact that there exists a formulation for gravity in four dimensions with manifest Ehlers symmetry suggests that a nonlinear extension of the duality-invariant action in [20] might lie within reach.

The prepotential formalism has been instrumental in constructing several first-order Hamiltonian actions for self-dual fields, which include the free actions for $N=$ $(4,0)$ and $N=(3,1)$ supergravity in six dimensions $[22,23]$ to name a few. But the construction of interacting actions for these self-dual fields has been a longstanding problem, for there exists a plethora of no-go theorems that rule out such interactions on the grounds of Lorentz covariance and locality (see [24-27] for a nonexhaustive list). The light-cone formalism, on the contrary, violates manifest Lorentz covariance and allows for a unique type of nonlocality in the spatial coordinates. These key ingredients seem to do the trick for constructing a dualityinvariant formulation for gravity at least up to the second order in coupling constant. In light of these results, it would be interesting to see if the inclusion of certain elements of nonlocality in the prepotential formalism can help circumvent the aforementioned no-go results. Likewise, one could try to incorporate similar tools into the powerful framework of Becchi-Rouet-Stora-Tyutin cohomology for gauge theories $[28,29]$, in order to explore the notion of spatial nonlocality with greater mathematical rigor. It would be nice to further extend the light-cone analysis of gravity to establish links to other interesting symmetry-related directions [30].

\section{ACKNOWLEDGMENTS}

We thank Sudarshan Ananth and Lars Brink for insightful discussions and for helpful comments on the manuscript. We are grateful to Glenn Barnich, Marc Henneaux, and Stefan Prohazka for many stimulating discussions. This work was partially supported by a Marina Solvay Fellowship, by the ERC Advanced Grant "High-Spin-Grav", and by FNRSBelgium (convention FRFC PDR T.1025.14 and convention IISN 4.4503.15). We acknowledge the kind hospitality offered during the workshop "Higher spins and Holography" at the Erwin Schrödinger Institute in Vienna, where a part of this work was completed.

\section{APPENDIX A: GRAVITY IN THE LIGHT-CONE GUAGE}

In this appendix we briefly discuss the light-cone gaugefixing and perturbative expansion of the Einstein-Hilbert action in four dimensions.

The Einstein-Hilbert action on a Minkowski background reads

$$
S_{\mathrm{EH}}=\int d^{4} x \mathcal{L}=\frac{1}{2 \kappa^{2}} \int d^{4} x \sqrt{-g} \mathcal{R}
$$

with the corresponding field equation

$$
\mathcal{R}_{\mu \nu}-\frac{1}{2} g_{\mu \nu} \mathcal{R}=0
$$

where the symbols have their usual meaning. We now impose three of the four allowed gauge choices $[13,14]$

$$
g_{--}=g_{-i}=0, \quad i=1,2 .
$$

The rest of the metric components are parametrized as

$$
\begin{aligned}
g_{+-} & =-e^{\phi}, \\
g_{i j} & =e^{\psi} \gamma_{i j} .
\end{aligned}
$$

$\phi, \psi$ are real parameters and $\gamma^{i j}$ is a real, symmetric matrix with unit determinant. As a result of the light-cone gauge choice, some of the field equations do not involve time derivatives $\left(\partial_{+}\right)$and become constraint relations which can be solved to eliminate more d.o.f. from the theory. The $\mu=\nu=-$ equation from (A2) is such a constraint relation which gives

$2 \partial_{-} \phi \partial_{-} \psi-2 \partial_{-}^{2} \psi-\left(\partial_{-} \psi\right)^{2}+\frac{1}{2} \partial_{-} \gamma^{i j} \partial_{-} \gamma_{i j}=0$. 
We now make our fourth gauge choice

$$
\phi=\frac{\psi}{2},
$$

which allows us to solve for $\psi$ in terms of $\gamma_{i j}$. Similarly we can use the other constraint relations to eliminate all the unphysical d.o.f. and obtain the light-cone action for gravity in a closed form expression

$$
\begin{aligned}
S= & \frac{1}{2 \kappa^{2}} \int d^{4} x e^{\psi}\left(2 \partial_{+} \partial_{-} \phi+\partial_{+} \partial_{-} \psi-\frac{1}{2} \partial_{+} \gamma^{i j} \partial_{-} \gamma_{i j}\right) \\
& -e^{\phi} \gamma^{i j}\left(\partial_{i} \partial_{j} \phi+\frac{1}{2} \partial_{i} \phi \partial_{j} \phi-\partial_{i} \phi \partial_{j} \psi\right. \\
& \left.-\frac{1}{4} \partial_{i} \gamma^{k l} \partial_{j} \gamma_{k l}+\frac{1}{2} \partial_{i} \gamma^{k l} \partial_{k} \gamma_{j l}\right) \\
& -\frac{1}{2} e^{\phi-2 \psi} \gamma^{i j} \frac{1}{\partial_{-}} R_{i} \frac{1}{\partial_{-}} R_{j},
\end{aligned}
$$

where

$$
\begin{aligned}
R_{i} \equiv & e^{\psi}\left(\frac{1}{2} \partial_{-} \gamma^{j k} \partial_{i} \gamma_{j k}-\partial_{-} \partial_{i} \phi-\partial_{-} \partial_{i} \psi+\partial_{i} \phi \partial_{-} \psi\right) \\
& +\partial_{k}\left(e^{\psi} \gamma^{j k} \partial_{-} \gamma_{i j}\right) .
\end{aligned}
$$

\section{Perturbative expansion}

We consider a perturbative expansion of the closed form action where we parametrize $\gamma_{i j}$ as [14]

$$
\gamma_{i j}=\left(e^{\kappa H}\right)_{i j}
$$

where $H$ is a traceless matrix since $\operatorname{det}\left(\gamma_{i j}\right)=1$. We choose

$$
H=\left(\begin{array}{cc}
h_{11} & h_{12} \\
h_{12} & -h_{11}
\end{array}\right),
$$

where $h_{11}$ and $h_{12}$ can be linearly combined to form the helicity states of the graviton

$$
h=\frac{\left(h_{11}+i h_{12}\right)}{\sqrt{2}}, \quad \bar{h}=\frac{\left(h_{11}-i h_{12}\right)}{\sqrt{2}} .
$$

The light-cone Lagrangian can then be expressed perturbatively in orders of the coupling constant $\kappa$. To the first order in $\kappa$, it reads

$$
\mathcal{L}_{2}=\frac{1}{2} \bar{h} \square h+2 \kappa \bar{h} \partial_{-}^{2}\left[-h \frac{\bar{\partial}^{2}}{\partial_{-}^{2}} h+\frac{\bar{\partial}}{\partial_{-}} h \frac{\bar{\partial}}{\partial_{-}} h\right]+\text { c.c. },
$$

with the light-cone d'Alembertian is defined as

$$
\square=2\left(\partial \bar{\partial}-\partial_{+} \partial_{-}\right)
$$

The Lagrangian to order $\kappa^{2}$ was presented in [14,15], while the order $\kappa^{3}$ corrections can be found in [31].

\section{APPENDIX B: THE FIELD REDEFINITION AS A CHANGE OF VARIABLES}

Another way to understand the nonlocal field redefinition is to consider the action functional in the Hamiltonian formulation given as

$\mathcal{S}(h, \bar{h})=\int d^{4} x\left(\pi_{h} \partial_{+} h+\pi_{\bar{h}} \partial_{+} \bar{h}-\mathcal{H}\right)=\int d^{4} x \mathcal{L}(h, \bar{h})$

with the corresponding path integral

$$
\mathcal{I}=\int[\mathcal{D} h][\mathcal{D} \bar{h}] e^{i \mathcal{S}(h, \bar{h})} .
$$

We can similarly define an action with the new fields

$$
\mathcal{S}(C, \bar{C})=\int d^{4} x \mathcal{L}^{\prime}(C, \bar{C}) .
$$

We can argue that the field redefinition amounts to a change of variables in the path integral with the action $S(h, \bar{h})$ replaced by $S^{\prime}(C, \bar{C})$, if the integration measure $[\mathcal{D} \ldots]$ remains invariant under the transformation. Under a general transformation, the integration measure changes as

$$
\int[\mathcal{D} h][\mathcal{D} \bar{h}] \rightarrow \int(\operatorname{det} J)[\mathcal{D} C][\mathcal{D} \bar{C}],
$$

where $J$ stands for the Jacobian of the transformation.

In our perturbative approach, the field redefinition is an expansion in orders of $\kappa$, i.e., $h=C+\kappa(\cdots)$. Hence, it easily follows that $\operatorname{det} J=1+\mathcal{O}(\kappa)$ in our case. Therefore, at the lowest order the path integral in terms of $C, \bar{C}$ reads

$$
\mathcal{I}^{\prime}=\int[\mathcal{D} C][\mathcal{D} \bar{C}] e^{i \mathcal{S}(C, \bar{C})},
$$

which is simply a change of variables in (B2). This essentially means both the path integrals $\mathcal{I}$ and $\mathcal{I}^{\prime}$ describe the same theory and will give rise to the same correlation functions or scattering amplitudes. 
[1] J. Ehlers, Konstruktionen und Charakterisierung von Losungen der Einsteinschen Gravitationsfeldgleichungen, Dissertation, Hamburg University, 1957.

[2] R. P. Geroch, A method for generating solutions of Einstein's equations, J. Math. Phys. (N.Y.) 12, 918 (1971).

[3] E. Cremmer and B. Julia, The $\mathrm{SO}(8)$ supergravity, Nucl. Phys. B159, 141 (1979).

[4] N. Marcus and J.H. Schwarz, Three-dimensional supergravity theories, Nucl. Phys. B228, 145 (1983).

[5] T. Damour, M. Henneaux, and H. Nicolai, E(10) and a "Small Tension Expansion" of M Theory, Phys. Rev. Lett. 89, 221601 (2002).

[6] O. Hohm and H. Samtleben, U-duality covariant gravity, J. High Energy Phys. 09 (2013) 080.

[7] O. Hohm and H. Samtleben, Exceptional Form of $D=11$ Supergravity, Phys. Rev. Lett. 111, 231601 (2013).

[8] O. Hohm and H. Samtleben, Exceptional field theory. II. $\mathrm{E}_{7(7)}$, Phys. Rev. D 89, 066017 (2014).

[9] O. Hohm and H. Samtleben, Exceptional field theory. III. $\mathrm{E}_{8(8)}$, Phys. Rev. D 90, 066002 (2014).

[10] S. Ananth, L. Brink, and S. Majumdar, Exceptional versus super-Poincaré algebra as the defining symmetry of maximal supergravity, J. High Energy Phys. 03 (2016) 051.

[11] S. Ananth, L. Brink, and S. Majumdar, $\mathrm{E}_{8}$ in $\mathcal{N}=8$ supergravity in four dimensions, J. High Energy Phys. 01 (2018) 024.

[12] S. Ananth, L. Brink, and S. Majumdar, A hidden symmetry in quantum gravity, J. High Energy Phys. 11 (2018) 078.

[13] J. Scherk and J. H. Schwarz, Gravitation in the light-cone gauge, Gen. Relativ. Gravit. 6, 537 (1975).

[14] I. Bengtsson, M. Cederwall, and O. Lindgren, Light Cone Actions For Gravity And Higher Spins: Some Further Results, Goeteborg University 83-55 (1984), p. 22.

[15] S. Ananth and S. Theisen, KLT relations from the EinsteinHilbert Lagrangian, Phys. Lett. B 652, 128 (2007).

[16] S. Ananth, L. Brink, and P. Ramond, Oxidizing superYangMills from $(N=4, d=4)$ to $(N=1, d=10)$, J. High Energy Phys. 07 (2004) 082.
[17] S. Ananth, L. Brink, S. Majumdar, M. Mali, and N. Shah, Gravitation and quadratic forms, J. High Energy Phys. 03 (2017) 169.

[18] S. Mandelstam, Light cone superspace and the ultraviolet finiteness of the $N=4$ model, Nucl. Phys. B213, 149 (1983).

[19] A. Gorsky and A. Rosly, From Yang-Mills Lagrangian to MHV diagrams, J. High Energy Phys. 01 (2006) 101; P. Mansfield, The Lagrangian origin of MHV rules, J. High Energy Phys. 03 (2006) 037.

[20] M. Henneaux and C. Teitelboim, Duality in linearized gravity, Phys. Rev. D 71, 024018 (2005).

[21] R. A. Matzner and C. W. Misner, Gravitational field equations for sources with axial symmetry and angular momentum, Phys. Rev. 154, 1229 (1967).

[22] M. Henneaux, V. Lekeu, and A. Leonard, The action of the (free) (4, 0)-theory, J. High Energy Phys. 01 (2018) 114.

[23] M. Henneaux, V. Lekeu, J. Matulich, and S. Prohazka, The action of the (free) $\mathcal{N}=(3,1)$ theory in six spacetime dimensions, J. High Energy Phys. 06 (2018) 057.

[24] X. Bekaert, N. Boulanger, and M. Henneaux, Consistent deformations of dual formulations of linearized gravity: A no-go result, Phys. Rev. D 67, 044010 (2003).

[25] X. Bekaert, N. Boulanger, and S. Cnockaert, No selfinteraction for two-column massless fields, J. Math. Phys. (N.Y.) 46, 012303 (2005).

[26] X. Bekaert, M. Henneaux, and A. Sevrin, Chiral forms and their deformations, Commun. Math. Phys. 224, 683 (2001).

[27] N. Boulanger and S. Cnockaert, Consistent deformations of [p,p] type gauge field theories, J. High Energy Phys. 03 (2004) 031.

[28] G. Barnich and M. Henneaux, Consistent couplings between fields with a gauge freedom and deformations of the master equation, Phys. Lett. B 311, 123 (1993).

[29] G. Barnich, F. Brandt, and M. Henneaux, Local BRST cohomology in gauge theories, Phys. Rep. 338, 439 (2000).

[30] A. Strominger, On BMS invariance of gravitational scattering, J. High Energy Phys. 07 (2014) 152.

[31] S. Ananth, The quintic interaction vertex in light-cone gravity, Phys. Lett. B 664, 219 (2008). 